\title{
Factores de riesgo asociados a hipoacusia neonatal en pacientes del cunero fisiológico
}

\section{Risk factors associated with neonatal hearing loss in patients in the physiological nursery}

\author{
Jonathan A. Ponce-Castell ${ }^{1,2 \star}$, Carolina Carreto-Espinosa ${ }^{1,2}$, Jose Iglesias-Leboreiro ${ }^{3,4}$, \\ Isabel Bernardez-Zapata ${ }^{3}$, Désirée Vidaña-Pérez ${ }^{5}$, Rubén Martínez-Casanova ${ }^{3}$ y Héctor L.G. Macías-Villa ${ }^{6}$

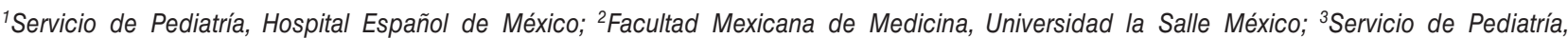 \\ Departamento de Neonatología, Hospital Español de México; ${ }^{4}$ Unidad de Cuidados Intensivos Neonatales; ${ }^{5}$ Ciencias de la Salud Reproductiva, \\ Instituto Nacional de Salud Pública; ${ }^{6}$ Departamento de Pediatría-Nefrología, Centro de Diagnóstico Ángeles. Ciudad de México, México
}

\section{Resumen}

\begin{abstract}
Antecedentes: La hipoacusia es una alteración física que en caso de no ser detectada a tiempo y de manera correcta causa afectaciones en lenguaje, habla y rendimiento escolar. En México se estima que cada año nacen entre 2,000 y 6,000 niños con sordera congénita y que 3 de cada 1,000 recién nacidos presenta hipoacusia. Objetivo: El objetivo del estudio fue analizar a los recién nacidos pretérmino tardío y de término mediante potenciales evocados auditivos de tallo cerebral como tamiz auditivo e identificar los factores de riesgo asociados a hipoacusia neonatal. Material y métodos: Se realizó un estudio transversal recopilando un total de 2,418 tamices auditivos del 1 de agosto de 2017 al 31 de mayo de 2019 . Se hicieron análisis descriptivos de la población de estudio evaluando sus características y se realizaron coeficientes de correlación. Para el análisis inferencial se corrieron modelos de regresión logística con la variable de presentar problemas auditivos como variable dependiente ajustados por sexo, semanas de gestación, tipo de parto, edad materna, índice de masa corporal (IMC) de la madre, comorbilidades de la madre e infección materna durante el embarazo. Resultados: Se encontró que pacientes con mayor edad gestacional tienen un $81 \%$ menor probabilidad de desarrollar hipoacusia (odds ratio [OR]: 0.19; $p=0.005)$. También encontramos que a mayor IMC materno el riesgo de presentar alteración auditiva incrementa 10 veces más (OR: 10.36; $p=0.025)$. Conclusiones: Es necesario llevar estudios diseñados específicamente para la evaluación de factores de riesgo de hipoacusia para poder prevenir y tratar a tiempo esta afección.
\end{abstract}

Palabras clave: Hipoacusia. Recién nacidos. Factores de riesgo.

\section{Abstract}

Background: Hearing loss is a physical alteration that, if is not detected on time and correctly, causes effects on language, speech and school performance. In Mexico, it is estimated that every year between 2,000 and 6,000 children are born with congenital deafness and that 3 of every 1,000 newborns have hearing loss. Objective: The main purpose of the study was to analyze late preterm and term newborns using brainstem auditory evoked potentials as an auditory screen and to identify risk factors associated with neonatal hearing loss. Material and methods: We performed a cross sectional study, collecting a total of 2,418 hearing screens from August 1, 2017 to May 31, 2019. Descriptive analysis of the study population was

Correspondencia:

*Jonathan A. Ponce-Castell

E-mail: dr.jonathan.ponce@gmail.com
Disponible en internet: 13-09-2021 Rev Hosp Jua Mex. 2021;88(3):130-135

www.revistahospitaljuarez.com 1405-9622/@ 2021 Sociedad Médico-Quirúrgica del Hospital Juárez de México, A.C. Publicado por Permanyer. Este es un artículo open access bajo la licencia CC BY-NC-ND (http://creativecommons.org/licenses/by-nc-nd/4.0/). 
performed evaluating its characteristics, as well as correlation coefficients. For the inferential analysis, logistic regression models were performed with the variable of having hearing problems as the dependent variable adjusted for sex, gestational age, type of delivery, maternal age, mother's BMI, mother's comorbidities, and maternal infection during pregnancy. Results: We found that patients with a higher gestational age have $81 \%$ lower probability to develop hearing loss (OR $0.19, p$-value $=0.005)$. We also found that a higher maternal BMI increases 10 times the risk of presenting hearing problems (OR 10.36, $p$-value $=0.025)$. Conclusions: It is necessary to make studies specifically designed for the evaluation of risk factors related to hearing loss to prevent and treat this condition on time.

Key words: Hearing loss. Newborn. Risk factors.

\section{Introducción}

La hipoacusia es una alteración física que en caso de no ser detectada a tiempo y de manera correcta causa afectaciones en lenguaje, habla y rendimiento escolar. Según la Organización Mundial de la Salud (OMS), la pérdida de la audición afecta al menos a 360 millones de personas en el mundo, de los cuales aproximadamente 32 millones son niños ${ }^{1}$. En México se estima que cada año nacen entre 2,000 y 6,000 niños con sordera congénita ${ }^{2}$ y que 3 de cada 1,000 neonatos presenta hipoacusia; esto se puede elevar hasta 4 de cada 1,000 cuando existen factores de riesgo? ${ }^{2}$.

Alrededor del $60 \%$ de las causas de hipoacusia en la niñez son prevenibles ${ }^{1}$. El $31 \%$ de las causas son infecciosas, el $17 \%$ están relacionadas con el parto y periodo prenatal, y el $4 \%$ con medicamentos ototóxi$\cos ^{1}$. Además, un gran porcentaje de la hipoacusia se considera de origen hereditario ${ }^{2}$. Otros factores de riesgo incluyen infección intrauterina y prematuridad ${ }^{1}$.

En la actualidad existen dos métodos usados como tamizaje para la detección de alteraciones en la audición: el primero son las emisiones otoacústicas (EOA) y el segundo los potenciales evocados auditivos de tallo cerebral (PEATC) ${ }^{3}$. Los PEATC han demostrado superioridad, ya que evalúan un trayecto más largo, lo que permite la identificación de alteraciones más profundas. Son capaces de diferenciar entre sordera de conducción, sordera neurosensorial o neurológica; o una lesión en la vía auditiva dentro del tallo cerebral ${ }^{4}$. Debido a esto, nuestro estudio tuvo como objetivo evaluar a todos los niños recién nacidos con el método de PEATC como estudio de inicio, con la finalidad de describir los casos de posible afectación auditiva que se presentan en el periodo neonatal y analizar posibles factores de riesgo involucrados para el desarrollo de hipoacusia neonatal. Actualmente son pocos los estudios que evalúan los factores de riesgo asociados en recién nacidos pretérmino tardío y de término y en todos los estudios solo se documenta el uso de EOA como método de detección oportuna, se reserva el uso de PEATC para pacientes que no pasaron de manera satisfactoria las primeras etapas de evaluación auditiva.

\section{Material y métodos}

Se realizó un estudio transversal que recopiló información de 2,434 tamices auditivos del 1 de agosto de 2017 al 31 de mayo de 2019. Los tamices fueron realizados a pacientes pretérmino tardío y de término en el cunero fisiológico del Hospital Español de México. Se usó la base de datos de la empresa Audi-k, que es la encargada de realizar los estudios de tamizaje en el hospital. Se incluyeron todos los pacientes vivos que nacieron dentro del Hospital Español, que tuvieron estancia en el cunero fisiológico, obtenidos por cesárea o parto natural y que tuvieran información completa de las variables, dando como muestra final un total de 2,418 recién nacidos. La medición del índice de masa corporal (IMC) se basó en el peso previo y durante el embarazo. Se llevaron a cabo análisis descriptivos de la población de estudio para evaluar sus características y se realizaron coeficientes de correlación. Para el análisis inferencial, se corrieron modelos de regresión logística con la variable de presentar problemas de audición como variable dependiente ajustados por sexo, semanas de gestación, tipo de parto, edad materna, IMC de la madre, comorbilidades de la madre e infección materna durante el parto. Todos los análisis se realizaron en STATA 14.2.

\section{Tamizaje}

El tamizaje en los recién nacidos se realizó utilizando el sistema Smart Screener Plus 2 con el software Smart USBlite. Este sistema evalúa los componentes cocleares y neurológicos en una sola prueba, además de evaluar simultáneamente ambos oídos ${ }^{5}$. El protocolo utilizado consta de tres etapas. En las dos primeras se realizan pruebas con el Smart Screener Plus 2, 
y en caso de no sea adecuada se pasa a la tercera etapa realizando PEATC de 7 a 15 días después. En caso de no pasar el examen se da referencia al servicio de audiología.

En la tercera etapa se solicita estudio de EOA para verificar el estado de la cóclea y además se realiza un estudio genético de la audición que investiga 15 factores genéticos y ambientales que causan pérdida auditiva hereditaria analizando los factores de mutaciones genéticas, paneles mitocondriales y detección de ADN para citomegalovirus.

\section{Variables}

La base de datos recogió variables del recién nacido y de características de la madre. Para los recién nacidos se contó con la variable categórica de sexo (femenino y masculino). Las semanas de gestación era una variable continua que categorizamos en pretérmino tardío (34 a 36.6 semanas) y productos de término ( 37 a 41.6 semanas). El tipo de obtención fue categorizado como parto y cesárea. La edad de la madre era una variable continua que categorizamos como madres menores de 35 y 35 o más años. El IMC materno fue categorizado como peso normal y exceso de peso. Además, se construyó una variable dicotómica de problemas de audición, la variable fue categorizada como sí/no, de acuerdo con la presencia o ausencia de problemas auditivos. Se recolectó información acerca de comorbilidades maternas como diabetes gestacional, hipertiroidismo e hipertensión, para la que se construyó una variable categórica: si presentó o no presentó comorbilidades. Por último, se preguntó si la madre sufrió algún tipo de infección durante el embarazo. El presente estudio estuvo exento de revisión por parte del comité de ética debido a que fue un análisis secundario de una base de datos y la base no contaba con información que permitiera identificar a los pacientes.

\section{Resultados}

Se estudió un grupo de 2,418 recién nacidos vivos, los cuales fueron evaluados para el tamiz auditivo neonatal con PEATC. La tabla 1 muestra las características de los recién nacidos y sus madres $(n=2,418)$. La muestra de recién nacidos tuvo una distribución equitativa de sexo ( $49.9 \%$ femenino y $50.1 \%$ masculino), con una media de 38 semanas de gestación y un $88.4 \%$ fueron de término. El $78.5 \%$ fueron obtenidos mediante cesárea. Durante el embarazo, el $50 \%$ de las madres presentaron sobrepeso $(50.2 \%)$, seguidas por
Tabla 1. Descripción de las características de la población $(n=2,418)$

\begin{tabular}{|c|c|}
\hline \multicolumn{2}{|c|}{ Características del recién nacido } \\
\hline $\begin{array}{l}\text { Sexo, } \% \\
\text { Femenino } \\
\text { Masculino } \\
\text { Semanas de gestación, } \mathrm{m} \pm \mathrm{DS}\end{array}$ & $\begin{array}{c}1,206(49.9 \%) \\
1,212(50.1 \%) \\
38 \pm 1.2\end{array}$ \\
\hline $\begin{array}{l}\text { Semanas de gestación, \% } \\
\text { Pretérmino tardío } \\
\text { Término }\end{array}$ & $\begin{array}{c}266(11.0 \%) \\
2,152(89.0 \%)\end{array}$ \\
\hline $\begin{array}{l}\text { Tipo de nacimiento, \% } \\
\text { Cesárea } \\
\text { Parto natural }\end{array}$ & $\begin{array}{c}1,897(78.5 \%) \\
521(21.5 \%)\end{array}$ \\
\hline $\begin{array}{l}\text { Características maternas } \\
\text { Edad materna, } \mathrm{m} \pm \mathrm{DS}\end{array}$ & $33.8 \pm 3.9$ \\
\hline $\begin{array}{l}\text { IMC, \% } \\
\text { Normal } \\
\text { Sobrepeso } \\
\text { Obesidad }\end{array}$ & $\begin{array}{c}1,087(44.9 \%) \\
1,212(50.2 \%) \\
119(4.9 \%)\end{array}$ \\
\hline $\begin{array}{l}\text { Comorbilidades* } n, \% \\
\text { No } \\
\text { Sí }\end{array}$ & $\begin{array}{l}1,053(43.5 \%) \\
1,365(56.5 \%)\end{array}$ \\
\hline $\begin{array}{l}\text { Infección materna, n,\% } \\
\text { No } \\
\text { Sí }\end{array}$ & $\begin{array}{c}1,975(81.7 \%) \\
443(18.3 \%)\end{array}$ \\
\hline
\end{tabular}

un $44.9 \%$ de madres con peso normal y solo el $5 \%$ de ellas con obesidad. El $56 \%$ de la muestra de las madres presentaron comorbilidades como diabetes gestacional, hipertensión e hipertiroidismo, por otro lado, únicamente el $18 \%$ de ellas presentó infección materna durante el embarazo.

No hubo diferencias estadísticamente significativas con respecto a la distribución de las calificaciones finales del tamiz auditivo con respecto a la distribución del sexo de los recién nacidos, el tipo de parto, ni en las comorbilidades ni infección materna. Sin embargo, las semanas de gestación, el IMC materno y la edad materna mostraron diferencias significativas (valor $\mathrm{p}$ : $0.10,0.025$ y 0.018 , respectivamente) (Tabla 2). Encontramos que los recién nacidos del sexo masculino tienen un $81 \%$ mayor probabilidad de tener un resultado de PEATC no satisfactorio. Además, observamos que los recién nacidos a término tienen un $81 \%$ menor probabilidad de tener un mal resultado de PEATC. Los recién nacidos de madres con sobrepeso u obesidad mostraron 10 veces mayor probabilidad de obtener un resultado negativo en su PEATC, siendo 
Tabla 2. Distribución de la muestra con respecto a la calificación final según los potenciales evocados auditivos de tallo cerebral (PEATC) del tamiz auditivo

\begin{tabular}{|c|c|c|c|c|c|}
\hline & \multicolumn{5}{|c|}{ Calificación final } \\
\hline & Pasa tamiz & Presunto positivo & Tamiz incompleto & Protocolo en proceso & valor $p^{*}$ \\
\hline $\begin{array}{l}\text { Sexo } \\
\text { Femenino } \\
\text { Masculino }\end{array}$ & $\begin{array}{l}1,201 \\
1,204\end{array}$ & $\begin{array}{l}1 \\
1\end{array}$ & $\begin{array}{l}1 \\
1\end{array}$ & $\begin{array}{l}4 \\
6\end{array}$ & 0.877 \\
\hline $\begin{array}{l}\text { Semanas de gestación } \\
\text { Pretérmino tardío } \\
\text { Término }\end{array}$ & $\begin{array}{c}261 \\
2,144\end{array}$ & $\begin{array}{l}0 \\
1\end{array}$ & $\begin{array}{l}0 \\
1\end{array}$ & $\begin{array}{l}5 \\
5\end{array}$ & 0.008 \\
\hline $\begin{array}{l}\text { Tipo de parto } \\
\text { Parto natural } \\
\text { Cesárea }\end{array}$ & $\begin{array}{c}519 \\
1,886\end{array}$ & $\begin{array}{l}0 \\
1\end{array}$ & $\begin{array}{l}1 \\
1\end{array}$ & $\begin{array}{l}1 \\
9\end{array}$ & 0.552 \\
\hline $\begin{array}{l}\text { IMC materno } \\
\text { Normal } \\
\text { Sobrepeso y obesidad }\end{array}$ & $\begin{array}{l}1,086 \\
1,319\end{array}$ & $\begin{array}{l}0 \\
1\end{array}$ & $\begin{array}{l}0 \\
2\end{array}$ & $\begin{array}{l}1 \\
9\end{array}$ & 0.025 \\
\hline $\begin{array}{l}\text { Comorbilidadest } \\
\text { Sin comorbilidades } \\
\text { Con comorbilidades }\end{array}$ & $\begin{array}{l}1,049 \\
1,356\end{array}$ & $\begin{array}{l}0 \\
1\end{array}$ & $\begin{array}{l}0 \\
2\end{array}$ & $\begin{array}{l}4 \\
6\end{array}$ & 0.718 \\
\hline $\begin{array}{l}\text { Infección materna } \\
\text { Sin infección } \\
\text { Con infección }\end{array}$ & $\begin{array}{c}1,964 \\
441\end{array}$ & $\begin{array}{l}1 \\
0\end{array}$ & $\begin{array}{l}2 \\
0\end{array}$ & $\begin{array}{l}8 \\
2\end{array}$ & 0.875 \\
\hline $\begin{array}{l}\text { Edad materna } \\
\quad<35 \\
\quad \geq 35\end{array}$ & $\begin{array}{c}1,337 \\
894\end{array}$ & $\begin{array}{l}0 \\
1\end{array}$ & $\begin{array}{l}0 \\
2\end{array}$ & $\begin{array}{l}9 \\
1\end{array}$ & 0.018 \\
\hline
\end{tabular}

*Prueba exacta de Fisher.

${ }^{\dagger}$ Comorbilidades: diabetes gestacional, hipertensión e hipotiroidismo.

IMC: índice de masa corporal.

estadísticamente significativo. Aquellos recién nacidos con madres que presentaron comorbilidades durante el embarazo como diabetes gestacional, hipertensión e hipotiroidismo, así como las madres que presentaron infección materna durante el embarazo, tuvieron un 96 y $49 \%$ mayor probabilidad de presentar resultados negativos en el PEATC, aunque estos resultados no fueron estadísticamente significativos (Tabla 3).

\section{Discusión}

El objetivo del estudio fue analizar a todos los recién nacidos pretérmino tardío y de término que fueron evaluados con PEATC como estudio de primera elección para el tamiz auditivo neonatal e identificar los factores de riesgo que están involucrados para el desarrollo de hipoacusia neonatal. Encontramos que los recién nacidos de término tuvieron significativamente un $81 \%$ menor probabilidad de desarrollar hipoacusia $(p=0.005)$. Este resultado es consistente con otros estudios que describen que la población más afectada son los pacientes prematuros y con factores de riesgo como infecciones, hiperbilirrubinemia y peso bajo al nacer. A pesar de que nuestro estudio no incluyó recién nacidos prematuros, es relevante observar que incluso en pretérmino las semanas de gestación juegan un papel importante en el desarrollo de alteraciones auditivas. Se ha observado que en pacientes menores de 37 semanas de gestación aumenta el riesgo de hipoacusia y más si el recién nacido tuvo bajo peso al nacer ${ }^{6}$.

Las madres que tenían un IMC elevado (sobrepeso u obesidad) tuvieron 10 veces más riesgo de tener hijos con hipoacusia, comparadas con aquellas que tuvieron un IMC normal $(p=0.025)$. Hasta donde sabemos no existen artículos que hayan explorado esta asociación en neonatos. Estudios realizados en población adolescente y adulta han encontrado que la obesidad podría tener influencia sobre la vía auditiva para generar hipoacusia, mediante la potencial alteración de la microvasculatura coclear ${ }^{7}$. Otro mecanismo biológico podría ser debido al incremento de ácidos grasos y triglicéridos 
Tabla 3. Regresión logística de los potenciales evocados auditivos de tallo cerebral (PEATC) y los factores asociados

\begin{tabular}{|c|c|c|}
\hline Variable & OR (IC 95\%) & valor $p$ \\
\hline $\begin{array}{l}\text { Sexo } \\
\text { Femenino } \\
\text { Masculino }\end{array}$ & $\begin{array}{c}\text { REF } \\
1.81(0.58-5.62)\end{array}$ & 0.304 \\
\hline $\begin{array}{l}\text { Semanas de gestación } \\
\text { Pretérmino tardío } \\
\text { Término }\end{array}$ & $\begin{array}{c}\text { REF } \\
0.19(0.06-0.68)\end{array}$ & 0.005 \\
\hline $\begin{array}{l}\text { Tipo de obtención } \\
\text { Cesárea } \\
\text { Parto natural }\end{array}$ & $\begin{array}{c}\text { REF } \\
1.01(0.21-4.74)\end{array}$ & 0.995 \\
\hline $\begin{array}{l}\text { IMC materno } \\
\text { Normal } \\
\text { Sobrepeso y obesidad }\end{array}$ & $\begin{array}{c}\text { REF } \\
10.36(1.31-80.36)\end{array}$ & 0.025 \\
\hline $\begin{array}{l}\text { Comorbilidades* } \\
\text { Sin comorbilidades } \\
\text { Con comorbilidades }\end{array}$ & $\begin{array}{c}\text { REF } \\
1.96(0.42-9.20)\end{array}$ & 0.393 \\
\hline $\begin{array}{l}\text { Infección materna } \\
\text { Sin infección } \\
\text { Con infección }\end{array}$ & $\begin{array}{c}\text { REF } \\
1.49(0.21-10.79)\end{array}$ & 0.687 \\
\hline $\begin{array}{l}\text { Edad materna } \\
\quad<35 \\
\quad \geq 35\end{array}$ & $\begin{array}{c}\text { REF } \\
0.69(0.21-2.28)\end{array}$ & 0.549 \\
\hline
\end{tabular}

*Comorbilidades: diabetes gestacional, hipertensión e hipotiroidismo. OR: odds ratio; IC: intervalo de confianza; IMC: índice de masa corporal REF: valor de referencia.

que generan estrés oxidativo y radicales libres que pueden alterar las estructuras auditivas ${ }^{8}$. Es posible que estos mecanismos puedan explicar parte de la relación encontrada en los neonatos, sin embargo, se necesitan mayores estudios para poder evaluar la asociación entre IMC materno elevado y la hipoacusia.

En nuestro estudio no encontramos asociación entre comorbilidades como diabetes gestacional, hipertensión gestacional e hipotiroidismo y la hipoacusia. Estos resultados contrastan con la literatura, ya que las enfermedades como hipertensión y diabetes mellitus se consideran factores de riesgo para el desarrollo de hipoacusia neonatal $1^{9,10}$. La falta de asociación podría ser explicada porque la mayor parte de nuestra población, a pesar de presentar comorbilidades, son pacientes que se encuentran con adecuado control y un buen seguimiento prenatal, esto puede generar una disminución en las consecuencias de las comorbilidades impidiendo afectar al producto.

Existen diversas limitaciones que deben de ser mencionadas. Primero, no contamos con información de variables relevantes como peso al nacer. Sin embargo, este estudio fue realizado en pacientes de término, lo que es una ventaja, ya que la mayor parte de la investigación de hipoacusia existente es en pacientes pretérmino ${ }^{11,12,13}$. Segundo, tuvimos poca variabilidad muestral, ya que solo contamos con pacientes recién nacidos del cunero fisiológico. Sin embargo, este estudio fue realizado utilizando los PEATC como método de medición, que han tenido una mejor viabilidad como estudio de tamizaje auditivo de primera elección ${ }^{8}$. Tercero, nuestros resultados no son generalizables a la población en general, sin embargo, podrían extrapolarse a poblaciones con características similares. Por último, debido a la naturaleza propia de los estudios transversales no podemos establecer causalidad entre el IMC elevado de la madre y la hipoacusia en el recién nacido, ni podemos descartar que exista confusión residual. Sin embargo, este resultado merece ser explorado debido a que la población femenina mexicana tiene una prevalencia combinada de más del $27 \%$ de sobrepeso y obesidad ${ }^{14}$, y este podría ser un factor importante para el desarrollo de diversas enfermedades y afecciones, entre ellas la hipoacusia neonatal.

\section{Conclusiones}

Los resultados de este estudio son novedosos, ya que se encontró que hay mayor riesgo de desarrollar hipoacusia en pacientes pretérmino tardío; otro resultado importante fue el aumento de riesgo en madres con IMC elevado. Se estima que en nuestro país la prevalencia de sobrepeso y obesidad en mujeres de edad reproductiva es del 13.2 y $14.1 \%$, respectivamente ${ }^{14}$. Estas comorbilidades pueden traer consecuencias físicas en el desarrollo de los recién nacidos. Es necesario llevar a cabo estudios diseñados específicamente para la evaluación de factores de riesgo de hipoacusia para poder prevenir y tratar a tiempo esta afección.

\section{Agradecimientos}

Los autores agradecen a las autoridades del Hospital Español de México, Programa Integral de Tamiz Metabólico e Intervención Temprana (Támi-K) y a la Universidad la Salle por las facilidades para poder llevar a cabo esta investigación.

\section{Financiamiento}

La presente investigación no ha recibido ninguna beca específica de agencias de los sectores públicos, comercial o sin ánimo de lucro. 


\section{Conflictos de intereses}

Los autores declaran que no existen conflicto de intereses en este estudio.

\section{Responsabilidades éticas}

Protección de personas y animales. Los autores declaran que para esta investigación no se han realizado experimentos en seres humanos ni en animales.

Confidencialidad de los datos. Los autores declaran que han seguido los protocolos de su centro de trabajo sobre la publicación de datos de pacientes

Derecho a la privacidad y consentimiento informado. Los autores han obtenido el consentimiento informado de los pacientes $y / 0$ sujetos referidos en el artículo.

\section{Bibliografía}

1. Organización Mundial de la Salud. Pérdida de audición en la niñez [Internet]. Organización Mundial de la Salud; 2016. Disponible en: http:// www.who.int/topics/deafness/WHD.pdf

2. González-Amaro C, Reyna-Barrientos $M$, Hernández-Sierra $F$, Suárez-Llanas B, Torres-Carreón F, Escalante-Padrón F. Hipoacusia en neonatos de alto riesgo. Pediatr de Mex. 2013;15(3):80-3.

3. Rado-Triveño J, Alen-Ayca J. Evaluación de las otoemisiones acústicas en relación a los potenciales evocados auditivos de tronco cerebral en niños. Rev Peru Med Exp Salud Publica. 2016;33(4):706-12.
4. Borrego C, Trujillo JM.. Potenciales evocados auditivos del tallo cerebral: registro, aplicaciones y datos normativos. Acta Med Colomb. 1985;10(1):114.

5. SmartScreener-Plus 2. Sistema de cribado auditivo infantil [Internet]. Intelligent Hearing Systems. Disponible en: http://www.insys.com/Brochures/BROCHURE SCREENER-PLUS SPA.pdf

6. Perez-Villegas R, Arriagada-Belmar M, Aviles-Muñoz M, Palma-Villegas J, Valenzuela-Maureira M. Factores maternos y perinatales asociados a hipoacusia: Serie de casos. Rev Colomb Obstet Ginecol. 2006;57(3):201-6.

7. Dhanda N, Taheri S. A narrative review of obesity and hearing loss. International Int J Obes (Lond). 2017;41(7):1066-73.

8. Granell J, Gavilanes J, Herrero J, Sánchez-Jara JL, Velasco MJ, Martín G. Cribado universal de la hipoacusia neonatal: ¿es más eficiente con potenciales evocados auditivos que con emisiones otoacústicas? Acta Otorrinolaringol (English Ed). 2008;59(4):170-5.

9. Hess CJ. Causas de hipoacusia sensorioneural. Rev Med Clin Las Condes [Internet]. 2003;14(1). Disponible en: http://www.clc.cl/clcprod/media/ contenidos/pdf/MED_14_4/CausasHipoacusiaSensorioneural.pdf

10. Trapero Dominguez M. Análisis descriptivo de los factores de riesgo en la hipoacusia infantil [tesis doctoral en Internet]. [Málaga]: Universidad de Málaga; 2015 [citado: 24 de diciembre de 2020]. Disponible en: https:// riuma.uma.es/xmlui/bitstream/handle/10630/14037/TD_TRAPERO_DOMINGUEZ_Maria_del_Carmen.pdf?sequence $=1$

11. Carranza Alva CA, Toral Martiñón R, Shkurovich Bialik $P$, Schabes Rostenberg M. Resultados del diagnóstico temprano de sordera en recién nacidos sin factores de riesgo. An Med (Mex) 2016;61(2):93-7.

12. Venegas Andrade A, Tello Valdés CA, Iglesias Leboreiro J, Bernárdez Zapata I, Cuevas Rivas AP, Rodríguez Santaolaya P, et al. Alteraciones auditivas en neonatos pretérmino a su egreso de una unidad de cuidados intensivos neonatales. Acta Pediatr Mex. 2020:41(1):1-10.

13. Comisión para la detección precoz de la hipoacusia. Programa para la detección precoz, el tratamiento y la prevención de la hipoacusia infantil. An Esp Ped. 1999;51:336-44.

14. Shamah-Levy T, Vielma-Orozco E, Heredia-Hernández O, Romero-Martínez M, Mojica-Cuevas J, Cuevas-Nasu L, et al. Encuesta Nacional de Salud y Nutrición 2018-19: Resultados Nacionales. Cuernavaca, México: Instituto Nacional de Salud Pública, 2020. Disponible en https://ensanut. insp.mx/encuestas/ensanut2018/doctos/informes/ensanut_2018_informe_final.pdf 